\title{
Extraction of synaptic input properties in vivo
}

\author{
Paolo Puggioni ${ }^{1,2}$, Marta Jelitai $^{3,4}$, Ian Duguid ${ }^{3}$ and Mark C.W. van Rossum²
}

May 6, 2016

\footnotetext{
${ }^{1}$ Neuroinformatics Doctoral training Centre, School of Informatics, University of Edinburgh

${ }^{2}$ Institute for Adaptive and Neural Computation, School of Informatics, University of Edinburgh, 10 Crichton Street, Edinburgh EH8 9AB, UK

${ }^{3}$ Centre for Integrative Physiology, University of Edinburgh, Hugh Robson Building, Edinburgh EH8 9XD, UK

${ }^{4}$ Presently at Institute of Experimental Medicine of the Hungarian Academy of Sciences, Budapest, Hungary.
}

\begin{abstract}
Knowledge of synaptic input is crucial to understand synaptic integration and ultimately neural function. However, in vivo the rates at which synaptic inputs arrive are high, that it is typically impossible to detect single events. We show here that it is nevertheless possible to extract the properties of the events, and particular to extract the event rate, the synaptic time-constants, and the properties of the event size distribution from in vivo voltage-clamp recordings. Applied to cerebellar interneurons our method reveals that the synaptic input rate increases from $600 \mathrm{~Hz}$ during rest to $1000 \mathrm{~Hz}$ during locomotion, while the amplitude and shape of the synaptic events are unaffected by this state change. This method thus complements existing methods to measure neural function in vivo.
\end{abstract}

\section{Significance Statement}

Neurons in vivo typically receive thousands of synaptic events per second. While methods have been developed to measure the total synaptic current that results from these events, extraction of the constituent events has proven very difficult given their high degree of overlap. To resolve this, we introduce a probabilistic method that extracts the statistics of synaptic event amplitudes and their frequency from voltage clamp recordings, which is then applied to recordings from cerebellar interneurons. With this method it becomes possible to better understand synaptic input and how it changes with behavioral state. 


\section{Introduction}

Neurons typically receive a barrage of thousands of excitatory and inhibitory events per second. As these inputs determine to a large extent the spiking activity of the neuron, it is important to know the properties of synaptic input and how it changes, for example, with behavioral state (e.g. sleep, attention, locomotion), with plasticity, or with homoeostasis. Consider a neuron receiving synaptic input while the total current is being measured in voltage-clamp, Fig.1a. While in vitro, or in cases where activity is artificially lowered, individual excitatory and inhibitory inputs can be resolved (top), in vivo the rates are typically so high that this is impossible. Instead, the total synaptic current trace is wildly fluctuating and single event extraction methods will fail.

Nevertheless, information can still be extracted from the statistical properties of the recorded in vivo currents. This study is based on the fact that although individual synaptic events might not be distinguishable in the observed current trace, the trace will still bear signatures of the underlying events. Intuitively, the mean current should be proportional to the product of the synaptic event size and the total event frequency. But it is possible to extract other information as well. For instance, when the synaptic events have short time-constants, the observed current trace will have more high frequency content than when the synaptic time-constants are slow. Similarly, when the input is composed of many small events, the variance of the current trace will be smaller than when it is composed of a few large events. Here we introduce a method that aims to infer the event rate, synaptic time-constants, and distribution of synaptic event amplitudes from the power spectral density and statistical moments of the observed current trace.

We applied our method to voltage-clamp traces of electrotonically compact interneurons recorded in the cerebellum of awake mice. We find that during voluntary locomotion, the excitatory input rate increases from 600 to $1000 \mathrm{~Hz}$, while the synaptic event amplitudes remain the same. Our method thus provides a novel way to resolve synaptic event properties in vivo.

\section{Methods}

We implemented the model in PyMC, a python package to perform Bayesian computation (Patil, Huard, and Fonnesbeck, 2010), using a Metropolis Hastings sampler, with normal proposal distribution and standard deviation in each dimension equal to 1 over the absolute value of the parameters. Usually, the auto-correlation of the chains was about $300-500$ samples and the burn-in phase was about 10 effective samples. To construct the posterior, we generated 150,00 samples yielding $\sim 400$ effective samples and assessed the mixing by using the Geweke method provided by the PyMC package. The computational analysis tools and data are available at www.to_be_announced.org.

To compare our method to traditional single event detection methods, we employed TaroTools, a freely available IgorPro package (see sites.google.com/site/tarotoolsregister/) to detect putative post-synaptic currents (PSCs).

The experimental data is described in detail elsewhere. Briefly, whole-cell patch clamp recordings of molecular layer interneurons were obtained from awake behaving but head-restrained mice at a depth 
a

Synaptic inputs

Arrival times

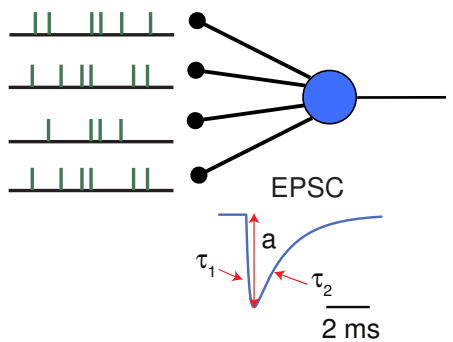

Voltage clamp trace
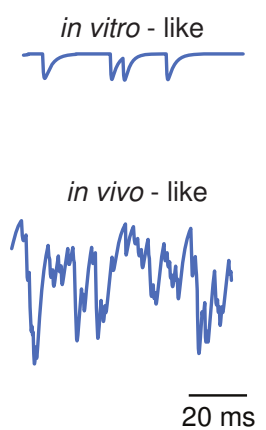

b Semi-automated single event analysis
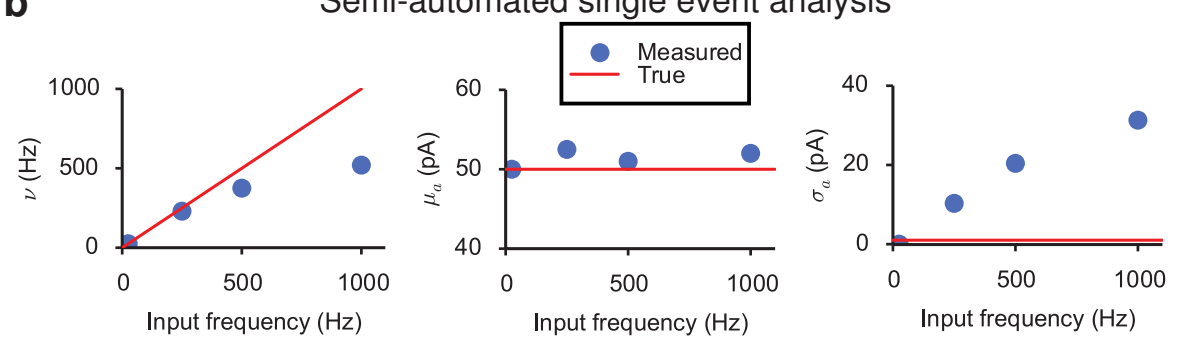

Figure 1. Inference of synaptic properties. a) A neuron receives input from a number of synapses under a Poisson rate assumption. The events have identical shape, but the amplitude $a$ varies between events. Right: For in vitro experiments synaptic events rates are typically low and the individual events can be extracted and quantified. However, for in vivo experiments, rates are high and individual events are not distinguishable. b) Analysis based on semi-automated single event extraction produces incorrect results when the total rate exceeds $500 \mathrm{HZ}$. From left to right: estimated event frequency, estimated mean event amplitude, estimated standard deviation of the event amplitude. Model parameters: $a_{k}=50 \mathrm{pA}$. rise-time $\tau_{1}=0.3 \mathrm{~ms}$ and the decay-time $\tau_{2}=2 \mathrm{~ms}$.

of 100-300 $\mu \mathrm{m}$ from the pial surface of the cerebellum, using a Multiclamp 700B amplifier (Molecular Devices, USA). The signal was filtered at $6-10 \mathrm{kHz}$ and acquired at $10-20 \mathrm{kHz}$ using PClamp 10 software in conjunction with a DigiData 1440 DAC interface (Molecular Devices). Patch pipettes (5-8 M $\Omega$ ) were filled with internal solution (285-295 mOsm) containing (in mM): $135 \mathrm{~K}$-gluconate, $7 \mathrm{KCl}, 10 \mathrm{HEPES}, 10$ sodium phosphocreatine, $2 \mathrm{MgATP}, 2 \mathrm{Na} 2 \mathrm{ATP}, 0.5 \mathrm{Na} 2 \mathrm{GTP}$ and $1 \mathrm{mg} / \mathrm{ml}$ biocytin (pH adjusted to 7.2 with $\mathrm{KOH}$ ). External solution contained (in $\mathrm{mM}$ ): $150 \mathrm{NaCl}, 2.5 \mathrm{KCl}, 10 \mathrm{HEPES}, 1.5 \mathrm{CaCl} 2,1 \mathrm{MgCl} 2$ (adjusted to $\mathrm{pH} 7.3$ with $\mathrm{NaOH}$ ).

To detect movement, the animals were filmed using a moderate frame rate digital camera (60 fps) synchronized with the electrophysiological recording. We defined a region of interest (ROI) covering the forepaws, trunk and face and calculated a motion index between successive frames (as in Schiemann et al., 2015). All movements (positioning, grooming and locomotion) were included. 


\section{Results}

A common method to extract synaptic properties is to identify and analyze isolated events from current traces, but in vivo this fails because the events will overlap, Fig.1a. To demonstrate the problem explicitly we simulated a neuron randomly receiving excitatory synaptic events (see below for model details). For illustration purposes we assume momentarily that the amplitude of all events is identical (50pA). From the total current recorded in voltage clamp we attempt to reconstruct the frequency of events and the distribution of their amplitudes.

We used single event dectection software (see Methods) to find putative post-synaptic currents (PSCs). At low input frequencies $(50 \mathrm{~Hz})$, most of PSCs were correctly identified and the resulting estimation of the synaptic input amplitude distribution was correct. However, at higher frequencies, when the event interval became shorter than the synaptic decay time, the event frequency was grossly underestimated and reached a plateau, Fig. 1b, left. At this point the individual EPSCs overlapped and became indistinguishable. The reason is that the most probable inter-time interval of a Poisson process (a common model for the inputs received by a neuron, but see Lindner, 2006) is zero. In addition, as a result of the overlap, the estimated PSC amplitude distribution had peaks at multiples of the original amplitude and the variance of the event amplitude was highly overestimated, Fig. 1b right. Finally, at high input frequencies the traces had to be manually post-processed to correct mistakes in event detection. This manual processing is time consuming - even an experienced researcher spent more than 1 hour to analyze a 10 second trace. Thus at high input frequencies single event analysis is not only incorrect, it is also time consuming.

\section{Generative model for the observed current trace}

Unlike the in vitro situation, the synaptic properties are not directly accessible from in vivo recordings. Instead, the data indirectly and stochastically reflects the synaptic properties. We therefore use a generative model to couple the data, in particular the statistics of the current trace, to the underlying synaptic properties. We define the generative model as follows: the synaptic inputs are assumed to arrive according to a Poisson process with a rate $\nu$, Fig.1a (also see Discussion). The synaptic events are modelled with a bi-exponential time-course as this can accurately fit most fast synapses (e.g. Roth and van Rossum, 2009)

$$
f(t)=\left(1-e^{-t / \tau_{1}}\right) e^{-t / \tau_{2}} \quad(t>0)
$$

While we initially assumed that all PSCs have the same time constants, the effect of heterogeneous time-constants is studied below. The total current is

$$
I(t)=\sum_{k=1}^{K} a_{k} f\left(t-t_{k}\right),
$$

where $t_{k}$ denotes the time of event $k$, and $a_{k}$ is the amplitude of that event. Unlike the schematic example above, the event amplitudes were drawn from a synaptic amplitude distribution $P(a)$ (with $a \geq 0$ ). This distribution captures the spread of amplitudes across the population of synapses, as well as variation in single synapse event amplitudes due to randomness and non-stationarities such as short-term plasticity. 
Although our method is general and not restricted to any specific distribution of synaptic amplitudes, we consider for concreteness the amplitudes to be distributed as either: 1) a log-normal distribution $(L N)$

$$
P(a)=\frac{1}{p_{2} \sqrt{2 \pi} a} \mathrm{e}^{-\frac{\left(\ln a-p_{1}\right)^{2}}{2 p_{p}^{2}}}
$$

with raw moments $\overline{a^{n}} \equiv \int_{0}^{\infty} P(a) a^{n} \mathrm{~d} a=e^{n p_{1}+n^{2} p_{2}^{2} / 2}$. Or, 2) a stretched exponential distribution (SE)

$$
P(a)=\frac{1}{p_{1} \Gamma\left(1+1 / p_{2}\right)} \mathrm{e}^{-\left(a / p_{1}\right)^{p_{2}}}
$$

with moments $\overline{a^{n}}=\frac{p_{1}^{n}}{p_{2}} \Gamma\left((1+n) / p_{2}\right) / \Gamma\left(1+1 / p_{2}\right)$ where $\Gamma(\cdot)$ is the Gamma function. Or, 3$)$ a zerotruncated-normal distribution $(T N)$

$$
P(a)=\frac{\phi\left(a / p_{2}+h\right)}{p_{2}[1-\Phi(h)]}
$$

where $h=-p_{1} / p_{2}$, and $\phi(\cdot)$ and $\Phi(\cdot)$ are the density of a normal distribution with zero-mean and unit variance and its cumulative. The mean $\mu_{a}=p_{1}+p_{2} \rho$ and variance $\sigma_{a}^{2}=p_{2}^{2}-p_{2}^{2}\left(\rho^{2}-\rho h\right)-\mu_{a}^{2}$, where $\rho=\phi(h) /[1-\Phi(h)]$ (for higher moments see Horrace, 2013).

The stretched exponential distribution has a maximum at zero amplitude, while the two other distributions have an adjustable mode that is non-zero, but differ in the heaviness of their tails. The $L N$ and $S E$ are heavy-tailed, while the $T N$ distribution is not. These three probability distributions are commonly used in the experimental and theoretical literature (Song et al., 2005; Barbour et al., 2007; Buzsáki and Mizuseki, 2014). Note that while conveniently all these distributions are characterized by the two parameters $p_{1}$ and $p_{2}$, which determine the mean and variance of the distribution, the parameters themselves are not comparable across distributions.

\section{Moments of the synaptic current}

Next, we calculated the current trace $I(t)$ that results from the random inputs. The statistics of the current follow from the distribution of synaptic event amplitudes and the time-course of the events according to Campbell's theorem (Rice, 1954; Bendat and Piersol, 1966; Ashmore and Falk, 1982). The cumulants $\kappa_{n}$ of the current probability distribution $P(I)$ follow from the event distribution and the synaptic time-course as

$$
\kappa_{n}=\nu \overline{a^{n}} \int_{0}^{\infty}[f(t)]^{n} d t
$$

In this equation the raw moments $\overline{a^{n}}$ of the synaptic event amplitude distribution $P(a)$, are given above for the different candidate distributions. Furthermore, for the bi-exponential synaptic kernel $f(t)$ (Eq. 1) the integrals are $\int_{0}^{\infty}[f(t)]^{n} d t=n ! \tau_{1} \Gamma\left(n \frac{\tau_{1}}{\tau_{2}}\right) / \Gamma\left(1+n+n \frac{\tau_{1}}{\tau_{2}}\right)$. Finally, the moments of the current trace $M_{I}$ 
are expressed in the cumulants $\kappa_{n}$. In practice we use the first four moments of the current distribution,

$$
\begin{aligned}
\mu_{I} & =\kappa_{1} \\
\sigma_{I} & =\sqrt{\kappa_{2}} \\
\operatorname{skew}(I) & =\kappa_{3} / \kappa_{2}^{3 / 2} \\
\operatorname{kurtosis}(I) & =\left(\kappa_{4}+3 \kappa_{2}\right) / \kappa_{2}^{2}-3 .
\end{aligned}
$$

We can thus express the statistical moments of the distribution of the observed current trace, Eq.7, in the underlying model.

\section{Power spectrum of the synaptic current}

Also the power spectral density (PSD) of the current $I(t)$ can be expressed in the model parameters (Puggioni, 2015). The current is the convolution a Poisson process, which has a flat power spectrum, with the synaptic kernel. As a result the PSD is the magnitude of the Fourier transform of the synaptic kernel and for non-zero frequencies equals

$$
\operatorname{PSD}(f)=2 \nu\left(\mu_{a}^{2}+\sigma_{a}^{2}\right) \frac{\tau_{2}^{4}}{\left(\tau_{1}+\tau_{2}\right)^{2}+\left(2 \pi f \tau_{2}\right)^{2}\left(2 \tau_{1}^{2}+2 \tau_{1} \tau_{2}+\tau_{2}^{2}\right)+\left(2 \pi f \tau_{2}\right)^{4} \tau_{1}^{2}} .
$$

Note that being a second order statistic, the PSD depends on the mean and variance of the amplitude distribution $P(a)$ only.

\section{Inference procedure}

Now that we have expressed both the PSD and the moments of the current distribution in the model parameters, one could proceed using classical fitting techniques, such as least square fitting, to find the synaptic parameters that best fit the data. However, we use a probabilistic approach that yields the distribution of parameters that best fit the data. A probabilistic approach is advantageous because 1) we expect strong correlations between the model parameters, 2) the probabilistic approach naturally yields the probability distribution of possible fit parameters, and 3) the probabilistic model is straightforwardly extended to include a variety of experimental effects that are crucial in describing the data.

However, before including these we first present an idealized model, which ignores some distortions typical of in vivo recordings. Fig. 2 shows the Bayesian network and the dependencies among the variables (nodes). The green nodes stand for variables that are measured directly from the data: the PSD and the first four moments of the current $M_{I}=\left[\mu_{I}, \sigma_{I}, \operatorname{skew}_{I}\right.$, kurtosis $\left._{I}\right]$. Together the data are succinctly denoted $\mathbf{D}$. The orange nodes represent variables that are to be inferred. The 5 parameters of the model are the rate $\nu$, the mean synaptic amplitude $\mu_{a}$, its variance $\sigma_{a}$, synaptic rise-time $\tau_{1}$ and decay time $\tau_{2}$, as well as the type of distribution $S_{a}$, Table 1 . The set of parameters is denoted $\theta$. 


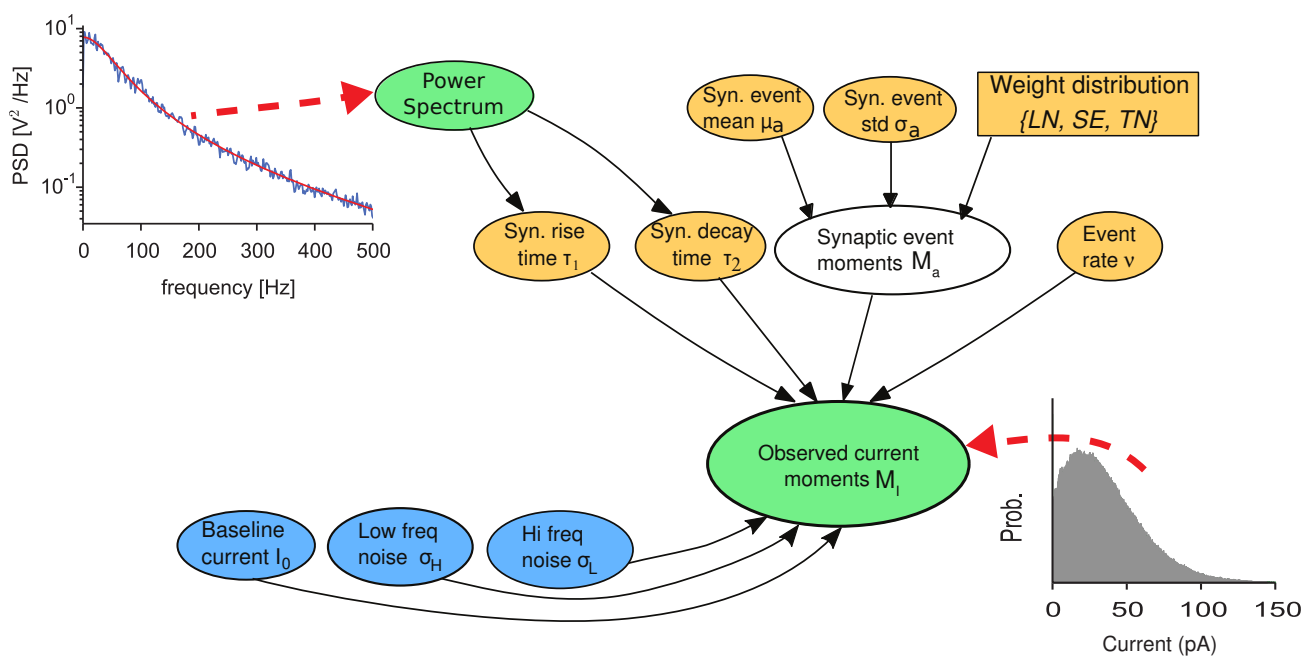

Figure 2. Bayesian network representing the dependencies between the variables. Orange nodes represent variables that have to be inferred from the data, green nodes stand for variables that are measured directly from the data. The blue nodes are additional contributions to the current in typical experiments. The top left graph shows the PSD fit (red line is the fit with Eq. 8) and the bottom right graph is the probability distribution of $I(t)$, used to calculate the observed moments $M_{I}$. All variables are described in Table 1.

Written formally, the joint probability of the Bayesian network in Fig. 2 is

$$
\begin{aligned}
P_{\text {joint }}(\theta, \mathbf{D})= & P\left(\tau_{1} \mid \mathrm{PSD}\right) P\left(\tau_{2} \mid \mathrm{PSD}\right) P\left(M_{a} \mid \mu_{a}, \sigma_{a}, S_{a}\right) \times \\
& P\left(\mu_{a}\right) P\left(\sigma_{a}\right) P(\nu) P\left(M_{I} \mid \tau_{1}, \tau_{2}, M_{a}, \nu\right)
\end{aligned}
$$

From this the parameter distribution given the data $P(\theta \mid \mathbf{D})$ follows as $P(\theta \mid \mathbf{D}) \propto P_{\text {joint }}(\theta, \mathbf{D})$. We now describe $P_{\text {joint }}$ and the probabilistic dependencies among the nodes term by term. The first two terms infer the values for the synaptic time-constants from the PSD. Since we cannot obtain an analytic expression of the likelihood of the PSD, we use empirical Bayes to set the prior on the time constants of the post-synaptic current (Casella, 1985). We fit Eq. 8 with a least square method to the PSD to find $\tau_{1}$ and $\tau_{2}$ (see top left inset in Fig. 2). Since we found the cross terms of the Hessian matrix between $\tau_{1}$ and $\tau_{2}$ to be very small $(<0.005)$, we model the time constants with independent Gaussian distributions with mean and variance given by the mean and the Hessian of the PSD fit. A common criticism of empirical Bayes is that it uses data for both prior and inference, thus double counting the data. Here however, the PSD data is used to set the prior on the time constants, but it is not used as evidence in the inference process, Fig. 2.

The next term in Eq. 9 is $P\left(M_{a} \mid \mu_{a}, \sigma_{a}, S_{a}\right)$. This is a deterministic function, because the moments of the synaptic amplitude distribution $M_{a}$ are fully determined by $\mu_{a}, \sigma_{a}$ and the type of amplitude distribution type, see Eqs. 3-5. The parameters $\mu_{a}, \sigma_{a}$ and $\nu$ are given uninformative uniform priors spanning a reasonable and positive range of values.

The final term in Eq. 9, the likelihood of the moments of the current $P\left(M_{I} \mid \tau_{1}, \tau_{2}, M_{a}, \nu\right)$ cannot be 


\begin{tabular}{|ll|}
\hline \multicolumn{1}{|c|}{ Parameter name } & \multicolumn{1}{c|}{ Description } \\
\hline$M_{I}=\left(\mu_{I}, \sigma_{I}\right.$, skew $_{I}$, kurtosis $\left._{I}\right)$ & $\begin{array}{l}\text { Measured data } \boldsymbol{D} \\
\text { Observed first four moments of the current } I(t) \\
P S D\end{array}$ \\
\hline & Power spectral density of $I(t)$ \\
& Parameter of idealized model \\
$\tau_{1}, \tau_{2}$ & Rise and decay time of the EPSCs \\
$S_{a}=\{L N, S E, T N\}$ & Synaptic amplitude distribution $=$ \\
& \{log-normal, stretched exponential, truncated normal $\}$ \\
$\mu_{a}, \sigma_{a}$ & Mean and std. of the amplitude distribution \\
$M_{a}$ & Moments of the synaptic amplitude distribution \\
$\nu$ & Frequency of synaptic inputs \\
\hline & Additional parameters of full model \\
$i_{0}$ & Voltage clamp baseline current \\
$\sigma_{H}$ & Std. of high frequency noise \\
$\sigma_{L}$ & Std. of low frequency fluctuations \\
\hline
\end{tabular}

Table 1. Description of the parameters and variables of the model.

calculated analytically. Although Eq. 6 gives the expected value, $M_{I}$ is a stochastic quantity that due to the Poisson process is different on each run and thus requires simulation. However, below we present a method to speed up its calculation.

\section{Inclusion of in vivo variability and other experimental confounds}

In vivo voltage clamp recordings show a number of effects that need to be included in the model via additional parameters. The first additional feature is the baseline current $\left(i_{0}\right)$ of the voltage clamp that has to be subtracted from the current. In in vitro situations one can estimate it by finding the baseline of the current trace, but due to the high rates this is challenging for in vivo recordings. Instead a prior probability of $P\left(i_{0}\right)$ was included. It was normally distributed with mean and variance estimated with an informed guess, reflecting the uncertainty in the value of $i_{0}$.

The second feature is the inclusion of high frequency noise coming from the recording set-up and for instance the stochastic opening and closing of ion channels. Its standard deviation $\sigma_{H}$ is measured experimentally and we model it as a zero mean Orstein-Uhlenbeck (OU) process

$$
d U_{H_{t}}=-\tau_{H} U_{H_{t}} d t+\sigma_{H} \sqrt{2 / \tau_{H}} d B_{t}
$$

where $B_{t}$ is a Wiener process and the cut-off frequency is $1 /\left(2 \pi \tau_{H}\right)=600 \mathrm{~Hz}$.

Finally we include low frequency fluctuations typically present in in vivo synaptic activity (e.g. Schiemann et al., 2015). We relax the constant rate assumption by adding a modulation term to the Poisson rate, which is modeled as an OU process with power $\sigma_{L}^{2}$ and cut-off frequency $f_{L}=1 /\left(2 \pi \tau_{L}\right)$ of $5 \mathrm{~Hz}$

$$
d U_{L_{t}}=-\tau_{L} U_{L_{t}} d t+\sigma_{L} \sqrt{2 / \tau_{L}} d B_{t}
$$




\section{Likelihood of the moments of the synaptic current}

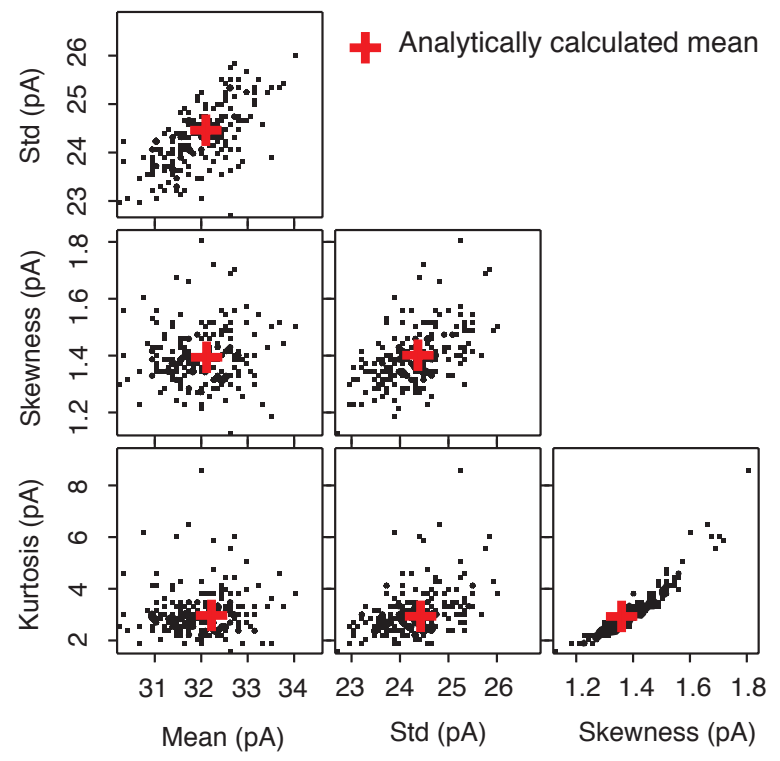

Figure 3. Likelihood of the moments of the synaptic current $P\left(M_{I} \mid \tau_{1}, \tau_{2}, M_{a}, \nu\right)$ as estimated by simulating the generative model multiple times with fixed parameters $\tau_{1}, \tau_{2}, M_{a}, \nu$. Red crosses represent the analytic predictions of the expected values of the $M_{I}$.

As a result in the expression for the PSD, Eq. 8, the rate $\nu$ is replaced by $\left(\nu+P S D_{O U}(f)\right)$, where the power spectrum of the OU process is given by $P S D_{O U}(f)=\sigma_{L}^{2} \tau_{L} /\left[1+\left(2 \pi f \tau_{L}\right)^{2}\right]$. To find the variance of this slow noise, we fit the PSD with Eq.8 in a range above $f_{L}$ and calculate its integral $\sigma_{t h}$ (the theoretical standard deviation of the modulation-free trace). Since the observed variance of the signal $\sigma_{o b s}^{2}$ is the sum of $\sigma_{t h}^{2}$ and $\sigma_{L}^{2}$ (the slow component is independent from the underlying process), it follows that $\sigma_{L}^{2}=\sigma_{o b s}^{2}-\sigma_{t h}^{2}$.

These three additional features are depicted by the blue nodes in Fig. 2. The joint probability becomes for the full model

$$
\begin{aligned}
P_{\text {joint }}= & P\left(\tau_{1} \mid \mathrm{PSD}\right) P\left(\tau_{2} \mid \mathrm{PSD}\right) P\left(M_{a} \mid \mu_{a}, \sigma_{a}, \mathrm{~S}_{\mathrm{a}}\right) P\left(\mu_{a}\right) P\left(\sigma_{a}\right) P(\nu) \times \\
& P\left(i_{0}\right) P\left(\sigma_{H}\right) P\left(\sigma_{L} \mid \mathrm{PSD}\right) P\left(M_{I} \mid \tau_{1}, \tau_{2}, M_{a}, \nu, i_{0}, \sigma_{H}, \sigma_{L}\right)
\end{aligned}
$$

\section{Description of the sampling algorithm}

In the Bayesian framework, the posterior probabilities of the parameters of the model can be estimated by sampling from $P_{\text {joint }}$, for instance using a suitable Markov chain Monte Carlo algorithm. However, this approach is very slow, because the likelihood does not have a closed form and has to be estimated with multiple simulations after each MCMC sample. As the estimation of the likelihood takes about 1 minute on a standard PC, a typical MCMC run of $\sim 100000$ samples would take approximately 2 months. 
We introduce a speed up that can be used whenever a likelihood can only be obtained by sampling from the generative model, but its means can be calculated analytically. The idea is to fit the likelihood with a kernel density estimate (KDE). Assuming that the shape of the likelihood does not depend much on the parameter values, the same KDE can be exploited to approximate the likelihood for different parameter values. As a result we can keep the shape fixed, but we translate it to a new location determined by the analytically calculated average moments of the likelihood. A thorough validation shows that the method correctly infers the parameters across a wide range of biologically plausible values (see below).

To perform the inference, we first initialize the parameters $\left\{\tau_{1}, \tau_{2}, M_{a}, \nu\right\}$ by Least-Square fitting Eq. 7 to the observed moments and Eq. 8 to the observed PSD. Next, we run the generative model multiple times to calculate the shape of $P\left(M_{I} \mid \tau_{1}, \tau_{2}, M_{a}, \nu\right)$ using an exponential KDE. Finally, during the main MCMC run where we sample $P_{\text {joint }}$, we keep the shape fixed but at each step we translate it to the location of the analytically calculated average moments (Eq. 6, red crosses in Fig 3).

\section{Validation on simulated data}
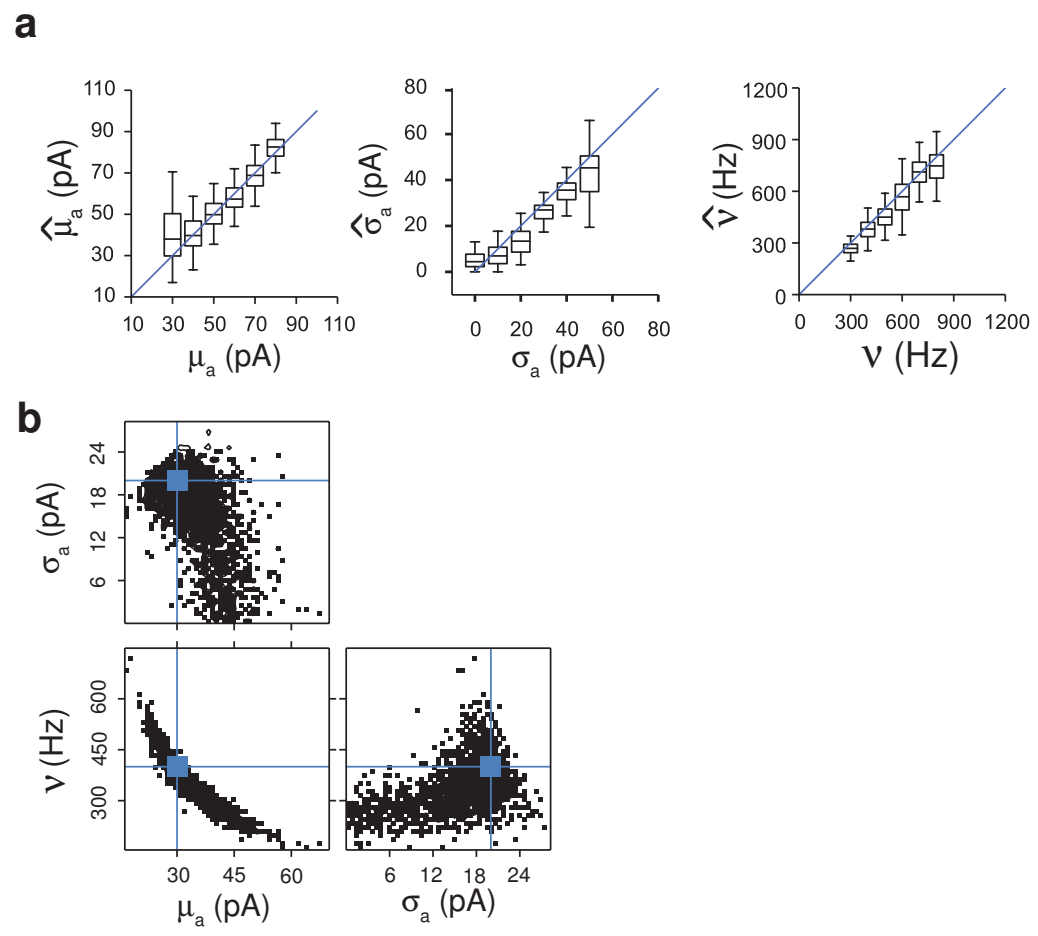

Figure 4. Validation of the method on simulated data.

a) The inference gives good results in a physiological range of parameters. True (x-axis) vs estimated parameters. The boxes represent the $33^{\text {rd }}$ and $66^{\text {th }}$ quantile of the distributions, the whiskers indicate the full range. The distribution of synaptic amplitudes was Log-Normal.

b) Inferred distribution of model parameters across multiple trials generated with fixed parameters (blue markers).

To validate the method we simulated 10s current traces with known parameters and we apply our 
inference method to recover their values. One parameter at a time was varied while the other parameters were set to a default value $\left(\mu_{a}=50 \mathrm{pA}, \sigma_{a}=40 \mathrm{pA}, \nu=700 \mathrm{~Hz}\right)$. We first assumed that the shape of the synaptic amplitude distribution ( $L N, S E$, or $T N)$ is a priori known. Fig. 4a compares the estimated parameters vs. their true value. The inference works well in a physiologically plausible range and the true value is almost always within the confidence interval. The largest error bars occur when either the mean event amplitude is small or the std dev. is large, i.e. the $\mathrm{CoV}$ is large.

The approach also yields the inferred joint distribution for a given parameter setting. The posterior distribution of the parameters contains the true values in the region of maximal density, Fig. 4b. Unlike single point estimates (e.g. maximum a posteriori, MAP estimates), one can also evaluate the dependencies between the parameters. In particular we observe a strong anti-correlation between event rate and event size (bottom left panel). In other words, the model compensates for changes in the rate by changing the estimate for the event size; their product is approximately invariant.

\section{Model selection}

Next, we tested whether the method is able to recover the correct amplitude distribution ( $L N, S E$, or, $T N$ ) when it is not known a priori. The Bayesian framework offers straightforward tools to assess the likelihood of a model, such as the Deviance Information Criterion (DIC) (Spiegelhalter et al., 2002). The higher is the DIC, the less likely is the model suitable to describe the data, and this would be the simplest way to choose the most likely distribution. However, the DIC value is a random variable that varies from trial to trial. Thus rather than selecting the lowest DIC, we use Bayesian model comparison based on the distribution of the DIC values. We generated 100 traces using a given amplitude distribution and run the inference algorithm assuming either $L N, S E$, or $T N$ amplitude distribution and we calculate the DIC for each mode, Fig. 5a. From the three DIC values of the three models $\mathrm{DIC}_{L N}, \mathrm{DIC}_{S E}$, and $\mathrm{DIC}_{T N}$ (corresponding to the $L N, S E$, and $T N$ model respectively) we calculate two quantities: $\Delta_{L T}=\mathrm{DIC}_{L N}-\mathrm{DIC}_{T N}$, and $\Delta_{L E}=\mathrm{DIC}_{L N}-\mathrm{DIC}_{S E}$. To find the most likely amplitude distribution, we apply Bayes theorem and calculate

$$
\begin{aligned}
P\left(X \mid \Delta_{L E}, \Delta_{L T}\right) & =\frac{P\left(\Delta_{L E}, \Delta_{L T} \mid X\right) P(X)}{\Sigma_{Y \in[L N, T N, S E]} P\left(\Delta_{L E}, \Delta_{L T} \mid Y\right) P(Y)}, \\
& =\frac{P\left(\Delta_{L E}, \Delta_{L T} \mid X\right)}{\Sigma_{Y \in[L N T N, S E]} P\left(\Delta_{L E}, \Delta_{L T} \mid Y\right)},
\end{aligned}
$$

where in the second line we assumed that each amplitude distribution is a priori equi-probable. Thus, for each point in the space $\left(\Delta_{L E}, \Delta_{L T}\right)$, we select the distribution which has the highest probability according to Eq.13, see Fig. 5b. This method is able to correctly identify the amplitude distribution with $\sim 90 \%$ accuracy, Fig. 5c.

\section{Robustness of method}

We examined the robustness of the method in a number of ways. First, we explored how the posterior of the parameters depends on the length of the trace. Longer traces should lead to less uncertainty and 

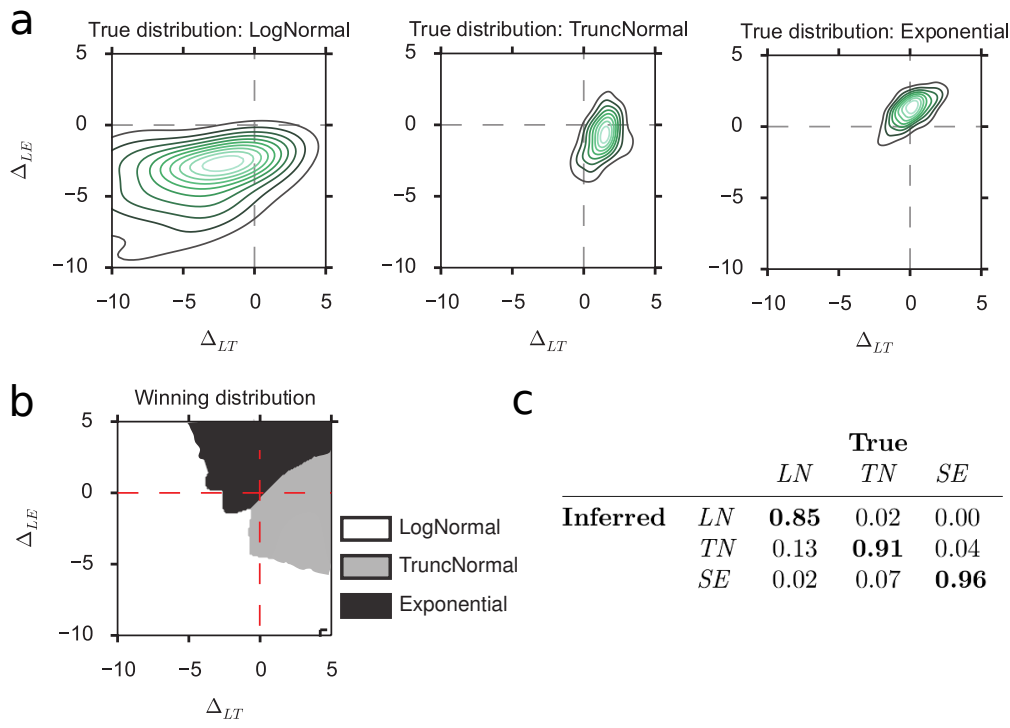

C

\begin{tabular}{llccl} 
& & \multicolumn{3}{c}{ True } \\
& & $L N$ & $T N$ & $S E$ \\
\hline Inferred & $L N$ & $\mathbf{0 . 8 5}$ & 0.02 & 0.00 \\
& $T N$ & 0.13 & $\mathbf{0 . 9 1}$ & 0.04 \\
& $S E$ & 0.02 & 0.07 & $\mathbf{0 . 9 6}$
\end{tabular}

Figure 5. Inference of the underlying weight distribution of simulated data. a) The distribution of DIC differences for the three simulation weight distributions. As the shapes of the distributions differ, we used Bayesian model selection. b) The resulting maximum likelihood solution that tells which underlying distribution is most likely. c) Performance of the algorithm to recover the correct weight distribution (expressed as fraction correct, based on 100 runs).

yield narrower, more precise distributions, because more statistics are collected. However, short intervals are preferable, because they allow the analysis of shorter periods in in vivo traces and allows one to see more rapid modulation in the synaptic inputs. Indeed, longer traces lead to less uncertainty on the parameters, Fig. 6a. The analysis shows that 10 second long recordings are in general enough to obtain a reasonable estimation of the parameters.

Next, we tested what happens when we introduce variability typical of in vivo recordings. Firstly, in vivo activity breaks the stationary assumption of the homogeneous Poisson model and inputs typically fluctuate on a slow time scale. To test the robustness of our model, we generate in vivo-like traces by adding an inhomogeneous component to the Poisson rate, modeled as a OU process with $5 \mathrm{~Hz}$ cutoff frequency. Again using simulated data, the model performs well even in presence of considerable fluctuations in the synaptic input rate, Fig. $6 \mathrm{~b}$.

Finally, in vivo PSCs rise- and decay-times might vary across synapses as different synapses may have different kinetic properties and may be subject to different amounts of dendritic filtering (Williams and Mitchell, 2008). To test whether our model performs well when the shape of the PSCs varies, the two time constants that determine the PSC shape were independently drawn from truncated normal distributions for each PSC. The model correctly extracted $\mu_{a}, \sigma_{a}$ and $\nu$ when the time-constants are heterogeneous, Fig. 6c. 

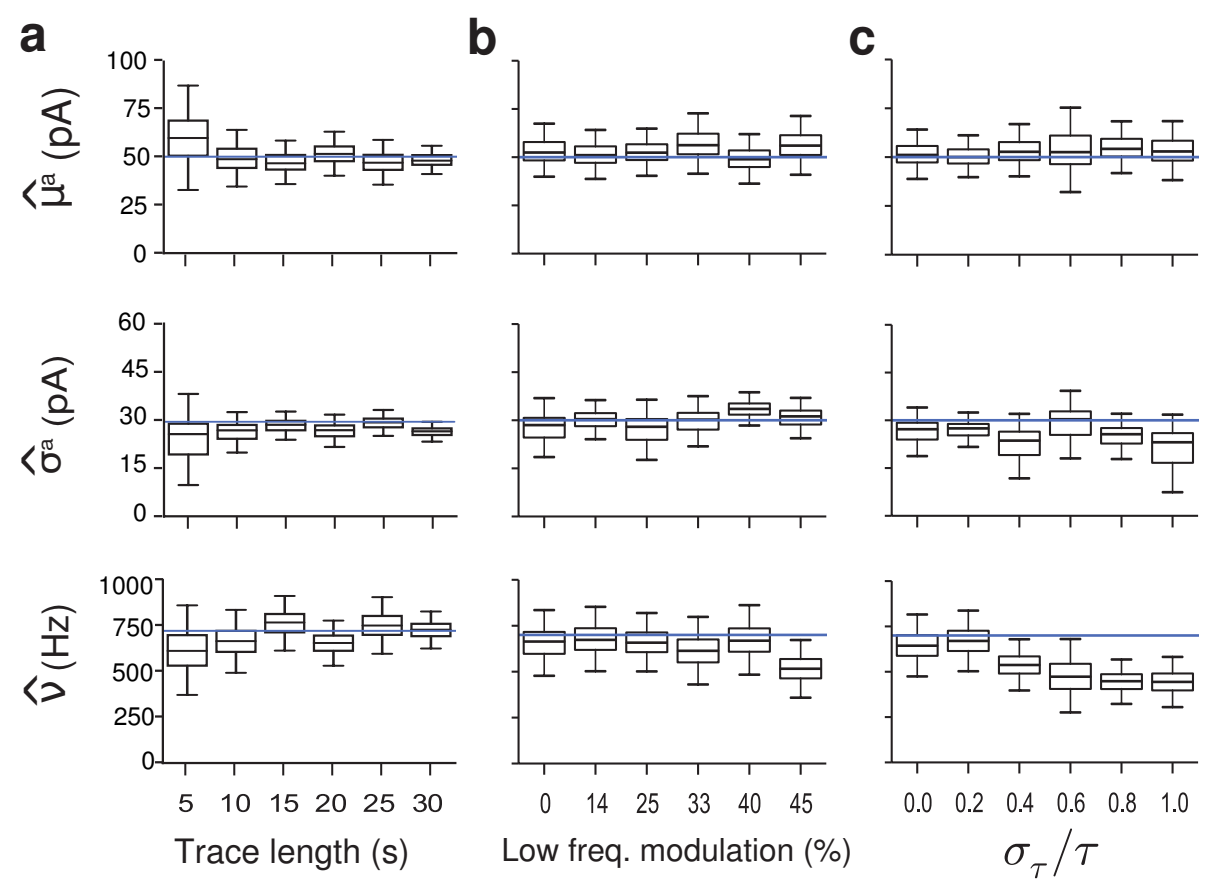

Figure 6. Robustness of inference demonstrated on simulated data. The traces were simulated with input amplitude drawn from a $\operatorname{LogNormal~distribution~}\left(\mu_{a}=50 \mathrm{pA}, \sigma_{a}=30 \mathrm{pA}\right)$ and $\nu=700 \mathrm{~Hz}$.

a) The estimates are robust using current traces of about 10s or longer. For shorter traces the inference is based on too little data and works less well. Top: mean single event amplitude $\mu_{a}$; middle: standard deviation of amplitude; bottom: event rate.

b) Robustness to in vivo variability when an inhomogeneous low frequency $(<5 \mathrm{~Hz})$ component is added to the Poisson rate. The parameters' estimation is plotted against the contribution (in percentage) of the low frequency modulation to the total standard deviation.

c) Robustness to heterogeneity in the synaptic time-constants as expressed in the CV of the rise- and decay-time constants.

\section{Inference method applied to cerebellar in vivo data}

We applied our inference method to in vivo recordings obtained from cerebellar interneurons. These neurons are ideal to test our method as they are electronically compact (Kondo and Marty, 1998). The voltage clamp held neurons at $-70 \mathrm{mV}$ to isolate excitatory inputs. The head-restrained mice displayed bouts of self-paced voluntary locomotion on a cylindrical treadmill, Fig. 7a. All traces $(n=8)$ were 90 seconds long and contained at least 10 seconds of movement. Locomotion modulates subthreshold and spiking activity in a large number of brain regions (Dombeck, Graziano, and Tank, 2009; Polack, Friedman, and Golshani, 2013; Schiemann et al., 2015). In cerebellar interneurons, locomotion is associated with increased excitatory input drive, Fig. 7b. In particular we were interested in what underlies this increased drive. For instance, it could be caused by increased frequency, increased amplitude as an effect of neuromodulation, or recruitment of a distinct set of synapses.

To apply our method we extracted the PSD and distribution from the current trace, Fig. 7b. The sub- 

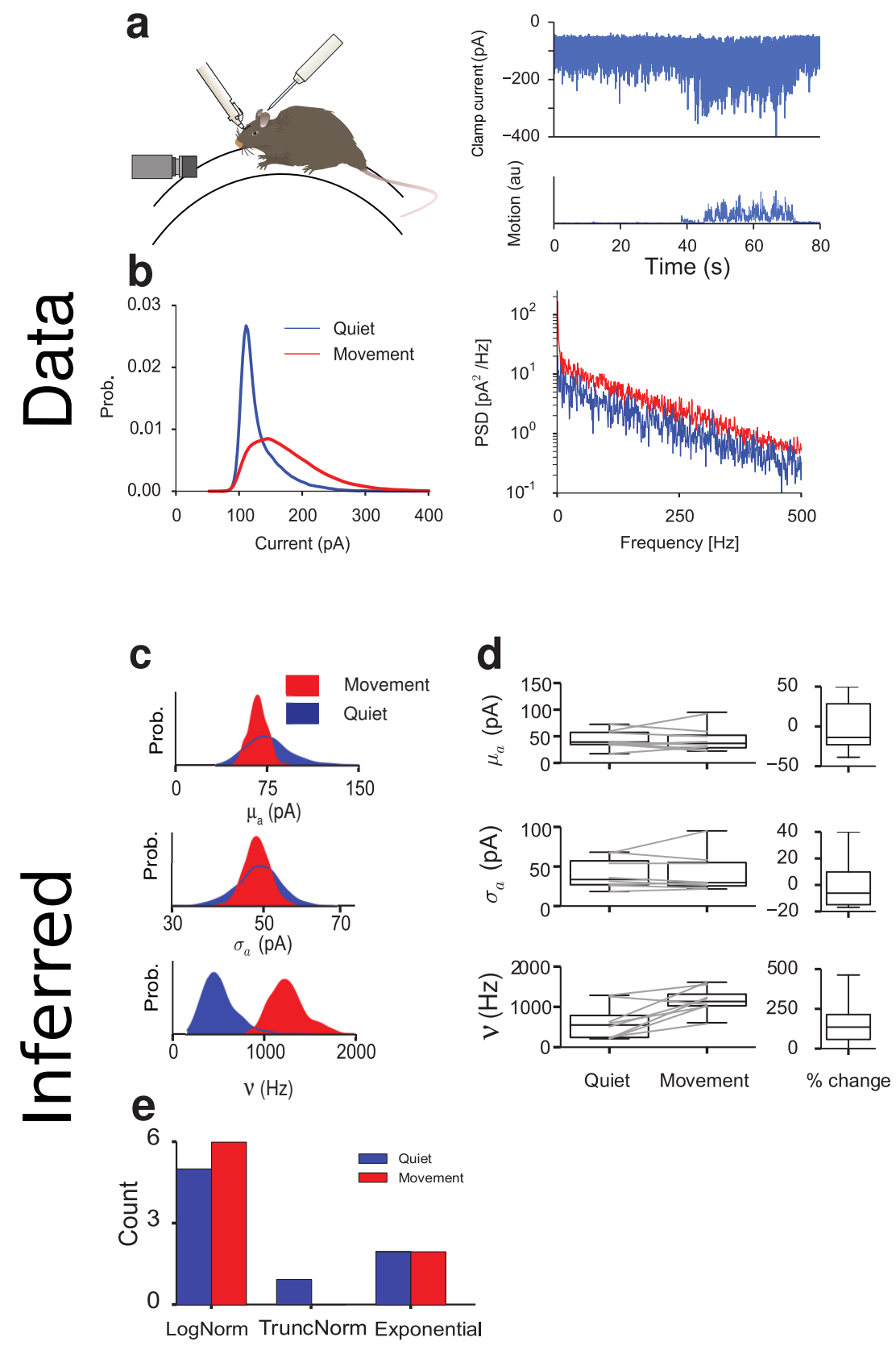

Figure 7. Analysis of in vivo voltage-clamp recordings.

a) Experimental setup: head-fixed awake mice, walking voluntarily on a wheel. Right: Voltage clamp current in cerebellar interneurons (top) and simultaneously recorded animal movement (bottom). Periods of movement are accompanied with an increased excitatory current in the neuron. b) Left: Observed current distribution in the moving and quiet periods. Note that due to the high input frequency, periods with zero current are very rare. Right: Samples of the recorded Power Spectral Density. c) Posterior distribution of the input parameters of a representative interneuron (under Log-Normal assumption, which was the most likely distribution for this neuron). d) Inference of the synaptic input parameters across 8 recordings displaying an increase in the input frequency during movement but not in the mean or variance of the event amplitude. e) Classification of the synaptic event amplitude distribution. In both conditions both Log-normal and Stretched exponential distributions were observed. The truncated normall tas inferred only once. Error bars denote the (min, $\max$ ) range, boxes the 25th-75th percentile, horizontal bar the median. 


\begin{tabular}{lccccccc}
\hline & \multicolumn{2}{c}{ Quiet } & \multicolumn{2}{c}{ Movement } & & power & power to \\
& mean & std err & mean & std err & p-value & of data & detect 10\% change \\
\hline$\tau_{1}$ & $0.27 \mathrm{~ms}$ & $0.03 \mathrm{~ms}$ & $0.28 \mathrm{~ms}$ & $0.03 \mathrm{~ms}$ & 0.24 & 0.15 & 0.61 \\
$\tau_{2}$ & $1.68 \mathrm{~ms}$ & $0.22 \mathrm{~ms}$ & $1.65 \mathrm{~ms}$ & $0.19 \mathrm{~ms}$ & 0.61 & 0.1 & 0.74 \\
$\mu_{a}$ & $42.8 \mathrm{pA}$ & $8.7 \mathrm{pA}$ & $43.2 \mathrm{pA}$ & $7.9 \mathrm{pA}$ & 1.00 & 0.04 & 0.83 \\
$\sigma_{a}$ & $31.3 \mathrm{pA}$ & $6.2 \mathrm{pA}$ & $31.0 \mathrm{pA}$ & $4.9 \mathrm{pA}$ & 0.86 & 0.04 & 0.42 \\
$\nu$ & $585 \mathrm{~Hz}$ & $153 \mathrm{~Hz}$ & $1006 \mathrm{~Hz}$ & $80 \mathrm{~Hz}$ & 0.03 & 0.93 & 0.07 \\
\hline
\end{tabular}

Table 2. Summary of the MAP values of the parameters estimated from $n=8$ in-vivo recordings.

sequent inference showed that the increase in excitatory synaptic current is associated with an increased input frequency, shown for a representative trace in Fig. 7c, bottom panel. However, movement did not lead to changes in the mean amplitude, or in the standard deviation of the synaptic amplitudes, Fig. 7c (top and middle panels). During movement the input frequency roughly doubles, from 585 to $1006 \mathrm{~Hz}$. The synaptic time constants found by fitting the power spectrum of the current, were $\tau_{1}=0.25 \pm 0.04$ ms and $\tau_{2}=1.56 \pm 0.21 \mathrm{~ms}$ (mean \pm standard error), comparable with the $20-80 \%$ rise time of $0.41 \pm$ $0.14 \mathrm{~ms}$ and the $1.85 \pm 0.52 \mathrm{~ms}$ decay reported in slice (Szapiro and Barbour, 2007).

Across the population the MAP estimates of $\mu_{a}, \sigma_{a}$ and $\nu$ during quiet wakefulness and movement show a similar pattern, Fig. $7 \mathrm{~d}$ and Table 2. Note that given the small changes between quiet and moving state, the power of the test calculated from the data is low, but $10 \%$ changes would be detected with high probability.

Next, we applied our inference method to each trace using the $L N$ (log-normal), SE (strechted exponential), and $T N$ (truncated normal) distribution and determined which synaptic amplitude distribution was the most likely. In general, we found that both during quiet periods and movement the most likely distributions were heavy-tailed being either LN or SE (with exponent on average 0.8, range 0.7 - 1.2), Fig. 7e. In particular, during active periods the $L N$ distribution (the most common) was significantly more likely than the $T N(\mathrm{p}=0.046)$, but the $S E$ distribution was not significantly less likely $(\mathrm{p}=0.37)$. Thus while this suggests that the distribution is strechted, the current data can not distinguish between the $L N$ and $S E$ types. Furthermore, we found no evidence for a change in the distribution shape between quiet and active period ( $L N, \mathrm{p}=0.78 ; S E, \mathrm{p}=0.96 ; T N, \mathrm{p}=0.71)$.

Finally, we compared our estimates to a standard single event extraction method (see Methods). Because the event extraction method fails at frequencies higher than $\sim 500$ inputs per second, the frequency of the synaptic inputs is underestimated by a factor two, due to the misclassification of overlapping events.

\section{Discussion}

In the last decade, numerous studies have been published using voltage-clamp data from anesthetized animals to investigate the contribution of excitation and inhibition to the $V_{m}$ dynamics, with recordings from auditory cortex (Wehr and Zador, 2003; Poo and Isaacson, 2009; Liu et al., 2010), visual cortex (Liu et al., 2010; Haider, Hausser, and Carandini, 2012), and pre-frontal cortex (Haider et al., 2006). 
However, in these experiments only the total excitatory or inhibitory contributions can be extracted, therefore they are unable to distinguish properties of single synapses and changes therein. We proposed a novel probabilistic method to infer the synaptic time-constants, the mean and variance of the synaptic event amplitude distribution, and the synaptic event rate from in vivo voltage-clamp traces. Moreover, the method accurately recovers the shape of the distribution of synaptic inputs. The inference is robust to slow fluctuations of synaptic input rate, experimental noise, and to heterogeneity in the time constants of the PSCs.

The extracted distribution reflects the amplitude of the events as received by the neuron. It therefore includes not only variations across synapses, but also variation due to synaptic unreliability and heterogeneity from effects like short-term synaptic plasticity (Szapiro and Barbour, 2007). Furthermore, the contribution of each synapse is weighted by its own input rate: synapses receiving inputs at higher rates will contribute more to the estimated amplitude distribution than synapses receiving low rates. Our method thus captures the effective distribution of synaptic inputs in an in vivo recording and thereby complements techniques that infer the amplitude distribution either anatomically from spine size or from paired recordings in vitro, and that are not weighted by the input rate.

Applied to voltage-clamp recordings from cerebellar interneurons of awake mice, we found that the excitatory synaptic amplitude distribution is either a stretched exponential or log-normal. This means that the probability for large events is larger than for a Gaussian with same mean and variance. Such heavy-tailed distributions have been observed in a number of systems (Sayer, Friedlander, and Redman, 1990; Song et al., 2005; Barbour et al., 2007; Ikegaya et al., 2013) and are believed to be an important characteristic of neural processing (Koulakov, Hromádka, and Zador, 2009; Roxin et al., 2011; Teramae, Tsubo, and Fukai, 2012). While any distribution can be tested (although for efficiency reasons the moments should ideally be available analytically), a future goal is to reconstruct the amplitude distribution directly, for instance by reconstructing it from it moments. However, there are currently no fully satisfactory mathematical methods to achieve this.

Furthermore we found no evidence that the synaptic amplitude distribution changes in these neurons when the animal is moving. Instead the increase of the excitatory current during movement is due to the higher frequency of the inputs. The most parsimonious explanation is that all inputs, big and small, increase their rates similarly during movement. However it is important to remember that the method is based on the ensemble of inputs. While our findings are inconsistent with a case where only large inputs become more active, and inconsistent with a case where all single synaptic events become stronger by, say, neuro-modulation, we can however not rule out that for instance a second population of inputs with an identical amplitude distribution becomes active during movement.

We summarize generalizations and restrictions of the method. First, as in most methods, the in vivo traces need to be stationary over a period long enough to accumulate sufficient statistics. The second assumption is that synaptic inputs are uncorrelated and follow a Poisson distribution. Experimental measurements of correlations in the brain are contradictory and largely depend on what time-scale is considered, reviewed in Cohen and Kohn (2011). Notably, slow correlations are visible in the PSD, adding a component with a different time-constant (Moreno-Bote, Renart, and Parga, 2008). When fitting the PSD of in vivo data, we observed a bump in activity in the low frequencies $(f<10 \mathrm{~Hz})$, 
that could correspond to spike correlations on time-scales $\gtrsim 15 \mathrm{~ms}$. Such correlations are included in our model. The method would not be able to identify spike-correlations on the order of the synaptic timeconstants $\left(\tau_{1}\right.$ and $\left.\tau_{2}\right)$, because they would contribute to the PSD in the same frequency range. However, it is generally believed that spike count correlations on a short time scale $(\sim 1-5 \mathrm{~ms})$ are small, normally < 0.03 (Smith and Kohn, 2008; Helias, Tetzlaff, and Diesmann, 2014; Grytskyy et al., 2013; Renart et al., 2010; Ecker et al., 2010), and thus the inference would likely still give correct results.

Finally, in these population measurements truly instantaneous correlations, where multiple events arrive simultaneously, can in principle never be distinguished from altered distributions. However, the error associated to this effect is likely limited. Consider a neuron that receives inputs of equal amplitude $a$ at a rate $\nu$. If the inputs have correlation $c=0.05$, it means that every 100 events, as a first approximation one will observe on average only 95 events, 90 of size $a$ and 5 of size $2 a$. In general, for a given correlation $c$, the observed frequency is $\nu_{\text {obs }}=\nu_{\text {true }}(1-c)$ and the observed average amplitude $a_{\text {obs }}=a_{\text {true }} /(1-c)$. Thus, even assuming $c=0.05$, the error in the estimate would be $\leq 5 \%$.

In principle, the method outlined here could be also applied to voltage-clamp recordings from pyramidal neurons in the cortex. However, the large size of their dendritic tree introduces space-clamp errors (Williams and Mitchell, 2008), so that the method estimates the net conductances at the soma.Earlier methods allow an estimation of the excitatory and inhibitory conductances using across trial average of current injections with different magnitude (Borg-Graham, Monier, and Frégnac, 1996; Anderson, Carandini, and Ferster, 2000; Wehr and Zador, 2003; Rudolph et al., 2004; Greenhill and Jones, 2007). More recently, conductances have been estimated from a single trace by applying a diverse range of probabilistic inference methods. In early studies the size of the excitatory and inhibitory inputs is assumed to be identical, fixed, and known a priori (Kobayashi, Shinomoto, and Lansky, 2011). Moreover, synaptic inputs were $\delta$-functions, with instantaneous rise and decay time and Poisson statistics. Some of the assumptions were relaxed in Paninski et al. (2012), where the number of inputs in a time window followed either an exponential or truncated Gaussian distribution, but the synaptic decay time constant has to be known a priori. Finally, Lankarany et al. (2013) further generalize the distribution of the number of inputs in a time window by making use of a mixture of Gaussians. This method allows a good estimation of the conductance traces even when the distribution of synaptic amplitudes has long tails. However, none of these methods estimate the frequency and amplitude distribution of the input events, but instead they recover the global excitatory and inhibitory conductances. As a result these techniques fail to distinguish between changes in input rate, and changes in synaptic strengths.

In summary, commonly used methods to analyse in vivo voltage clamp data can not infer the single event statistics at all or introduce large errors. Instead the proposed method represents an important step to extract such information from in vivo intracellular recordings.

\section{Acknowledgements}

We are grateful to M. Nolan, P. Latham, and L. Acerbi for helpful discussions. This work was supported by the Engineering and Physical Sciences Research Council Doctoral Training Centre in Neuroinformatics EP/F500385/1 and the Biotechnology and Biological Sciences Research Council BB/F529254/1 (PP), and 
a Wellcome Trust Career Development fellowship (WT086602MF) to ID.

\section{References}

Anderson, J. S., M. Carandini, and D. Ferster (2000). Orientation tuning of input conductance, excitation, and inhibition in cat primary visual cortex. J Neurophysiol 84(2): 909-26.

Ashmore, J. and G. Falk (1982). An analysis of voltage noise in rod bipolar cells of the dogfish retina. The Journal of physiology 332: 273.

Barbour, B., N. Brunel, V. Hakim, and J.-P. Nadal (2007). What can we learn from synaptic weight distributions? Trends Neurosci 30(12): 622-9.

Bendat, J. and A. Piersol (1966). Measurement and analysis of random data. Wiley.

Borg-Graham, L. J., C. Monier, and Y. Frégnac (1996). Voltage-clamp measurement of visually-evoked conductances with whole-cell patch recordings in primary visual cortex. J Physiol Paris 90(3-4): 185-8.

Buzsáki, G. and K. Mizuseki (2014). The log-dynamic brain: how skewed distributions affect network operations. Nat Rev Neurosci 15(4): 264-78.

Casella, G. (1985). An introduction to empirical bayes data analysis. The American Statistician 39(2): 83-87.

Cohen, M. R. and A. Kohn (2011). Measuring and interpreting neuronal correlations. Nat Neurosci 14(7): 811-9.

Dombeck, D. A., M. S. A. Graziano, and D. W. Tank (2009). Functional clustering of neurons in motor cortex determined by cellular resolution imaging in awake behaving mice. J Neurosci 29(44): 13751-60.

Ecker, A. S., P. Berens, G. A. Keliris, M. Bethge, N. K. Logothetis, and A. S. Tolias (2010). Decorrelated neuronal firing in cortical microcircuits. Science 327(5965): 584-7.

Greenhill, S. D. and R. S. G. Jones (2007). Simultaneous estimation of global background synaptic inhibition and excitation from membrane potential fluctuations in layer iii neurons of the rat entorhinal cortex in vitro. Neuroscience 147(4): 884-892.

Grytskyy, D., T. Tetzlaff, M. Diesmann, and M. Helias (2013). A unified view on weakly correlated recurrent networks. Front. Comput. Neurosci. 7: 131.

Haider, B., A. Duque, A. R. Hasenstaub, and D. A. McCormick (2006). Neocortical network activity in vivo is generated through a dynamic balance of excitation and inhibition. Journal of Neuroscience 26(17): 4535-45.

Haider, B., M. Hausser, and M. Carandini (2012). Inhibition dominates sensory responses in the awake cortex. Nature 493(7430): 97-100. 
Helias, M., T. Tetzlaff, and M. Diesmann (2014). The correlation structure of local neuronal networks intrinsically results from recurrent dynamics. PLoS Comput Biol 10(1): e1003428.

Horrace, W. (2013). Moments of the truncated normal distribution. J Prod Anal 43: 1-6.

Ikegaya, Y., T. Sasaki, D. Ishikawa, N. Honma, K. Tao, N. Takahashi, G. Minamisawa, S. Ujita, and N. Matsuki (2013). Interpyramid spike transmission stabilizes the sparseness of recurrent network activity. Cerebral Cortex 23(2): 293-304.

Kobayashi, R., S. Shinomoto, and P. Lansky (2011). Estimation of time-dependent input from neuronal membrane potential. Neural Comput 23(12): 3070-93.

Kondo, S. and A. Marty (1998). Synaptic currents at individual connections among stellate cells in rat cerebellar slices. J Physiol 509 ( Pt 1): 221-232.

Koulakov, A. A., T. Hromádka, and A. M. Zador (2009). Correlated connectivity and the distribution of firing rates in the neocortex. J Neurosci 29(12): 3685-94.

Lankarany, M., W.-P. Zhu, M. N. S. Swamy, and T. Toyoizumi (2013). Inferring trial-to-trial excitatory and inhibitory synaptic inputs from membrane potential using gaussian mixture kalman filtering. Front. Comput. Neurosci. 7: 109.

Lindner, B. (2006). Superposition of many independent spike trains is generally not a poisson process. Physical Review E 73(2): 022901.

Liu, B., P. Li, Y. J. Sun, Y. Li, L. I. Zhang, and H. W. Tao (2010). Intervening inhibition underlies simple-cell receptive field structure in visual cortex. Nat Neurosci 13(1): 89-96.

Moreno-Bote, R., A. Renart, and N. Parga (2008). Theory of input spike auto- and cross-correlations and their effect on the response of spiking neurons. Neural Comput 20(7): 1651-705.

Paninski, L., M. Vidne, B. Depasquale, and D. G. Ferreira (2012). Inferring synaptic inputs given a noisy voltage trace via sequential monte carlo methods. J Comput Neurosci 33(1): 1-19.

Patil, A., D. Huard, and C. Fonnesbeck (2010). Pymc: Bayesian stochastic modelling in python. Journal of statistical software $35(4): 1$.

Polack, P.-O., J. Friedman, and P. Golshani (2013). Cellular mechanisms of brain state-dependent gain modulation in visual cortex. Nature Neuroscience 16(9): 1331-9.

Poo, C. and J. S. Isaacson (2009). Odor representations in olfactory cortex: "sparse" coding, global inhibition, and oscillations. Neuron 62(6): 850-61.

Puggioni, P. (2015). Input-output transformations in the awak mouse brain using whole-cell recordings and probabilistic analysis. Ph.D. diss., School of Informatics, University of Edinburgh.

Renart, A., J. D. L. Rocha, P. Bartho, L. Hollender, N. Parga, A. Reyes, and K. D. Harris (2010). The asynchronous state in cortical circuits. Science 327(5965): 587-90. 
Rice, S. O. (1954). Mathematical analysis of random noise. In Wax, N., editor, Selected papers on noise and random processes. Dover, New York. reprinted from Bell System Technical Journal vols. 23 and 24.

Roth, A. and M. Rossum (2009). Modeling synapses. In Schutter, E. D., editor, Computational Modeling Methods for Neuroscientists. MIT Press.

Roxin, A., N. Brunel, D. Hansel, G. Mongillo, and C. V. Vreeswijk (2011). On the distribution of firing rates in networks of cortical neurons. J Neurosci 31(45): 16217-26.

Rudolph, M., Z. Piwkowska, M. Badoual, T. Bal, and A. Destexhe (2004). A method to estimate synaptic conductances from membrane potential fluctuations. J Neurophysiol 91(6): 2884-2896.

Sayer, R. J., M. J. Friedlander, and S. J. Redman (1990). The time course and amplitude of EPSPs evoked at synapses between pairs of CA3/CA1 neurons in the hippocampal slice. J Neurosci 10(3): 826-36.

Schiemann, J., P. Puggioni, J. Dacre, M. Pelko, A. Domanski, M. C. W. van Rossum, and I. Duguid (2015). Cellular mechanisms underlying behavioral state-dependent bidirectional modulation of motor cortex output. Cell Rep 11(8): 1319-1330.

Smith, M. A. and A. Kohn (2008). Spatial and temporal scales of neuronal correlation in primary visual cortex. Journal of Neuroscience 28(48): 12591-603.

Song, S., P. J. Sjöström, M. Reigl, S. Nelson, and D. B. Chklovskii (2005). Highly nonrandom features of synaptic connectivity in local cortical circuits. Plos Biol 3(3): e68.

Spiegelhalter, D., N. Best, B. Carlin, and A. V. D. Linde (2002). Bayesian measures of model complexity and fit. Journal of the Royal Statistical Society: Series B (Statistical Methodology) 64(4): 583-639.

Szapiro, G. and B. Barbour (2007). Multiple climbing fibers signal to molecular layer interneurons exclusively via glutamate spillover. Nat Neurosci 10(6): 735-42.

Teramae, J.-N., Y. Tsubo, and T. Fukai (2012). Optimal spike-based communication in excitable networks with strong-sparse and weak-dense links. Sci. Rep. 2: 485.

Wehr, M. and A. M. Zador (2003). Balanced inhibition underlies tuning and sharpens spike timing in auditory cortex. Nature 426(6965): 442-6.

Williams, S. R. and S. J. Mitchell (2008). Direct measurement of somatic voltage clamp errors in central neurons. Nat Neurosci 11(7): 790-8. 